\title{
Nuclear Symmetries of the similarity renormalization group for nuclear forces
}

\section{E. Ruiz Arriola*i}

Departamento de Física Atómica, Molecular y Nuclear and Instituto Carlos I de Física Teórica y Computacional. Universidad de Granada, E-18071 Granada, Spain.

E-mail: earriola@ugr.es

\section{S. Timóteo}

Faculdade de Tecnologia, Universidade Estadual de Campinas, 13484-332, Limeira - SP - Brazil

E-mail: varese@ft.unicamp.br

\section{S. Szpigel}

Centro de Ciências e Humanidades, Universidade Presbiteriana Mack enzie, 01302-907, São

Paulo - SP - Brazil

E-mail: szpigel@mackenzie.br

\begin{abstract}
We review the role played by long-distance symmetries within the context of the similarity renormalization group (SRG) approach. This is based on phase-shift-preserving continuous unitary transformations that evolve Hamiltonians with a cutoff on energy differences. We find that there is a SRG cutoff, $\lambda_{\text {Wigner }} \sim 3 \mathrm{fm}^{-1}$ for which almost perfect fulfillment of Wigner SU(4) symmetry is found at the two body level. This suggests to look for similar symmetry patterns for three- and four-body forces. We also analyze the impact of potentials based on Chiral Perturbation Theory in Nuclear Structure calculations.
\end{abstract}

The 7th International Workshop on Chiral Dynamics,

August 6 -10, 2012

Jefferson Lab, Newport News, Virginia, USA

${ }^{*}$ Speaker.

${ }^{\dagger}$ E.R.A. was supported by Spanish DGI (grant FIS2011-24149) and Junta de Andalucía (grant FQM225). S.S. was supported by Instituto Presbiteriano Mackenzie through Fundo Mackenzie de Pesquisa and FAPESP and V.S.T. by FAEPEX/PRP/UNICAMP and FAPESP. Computational power provided by FAPESP grants 2011/18211-2 and 2010/50646-6. 


\section{Introduction}

The use of effective interactions in Nuclear Physics has been the traditional procedure to sidestep the short-distance correlations triggered by the hard core of the NN potential below relative distances of about half a fermi [1,2]. While this is an acceptable requirement, we take the viewpoint that any sensible definition of an effective interaction should also unveil hidden symmetries so that they become manifest and can be exploited in the solution of the Nuclear Many Body Problem.

One outstanding and time honoured symmetry was suggested by Wigner in 1937 [3] to study nuclear specroscopy and the corresponding SU(4) spin-flavour symmetry group is generated by the Lie algebra of isospin $T^{a}$, spin $S^{i}$ and Gamow-Teller $G^{i a}$ generators in terms of the one particle spin $\sigma_{A}^{i}$ and isospin $\tau_{A}^{a}$ Pauli matrices,

$$
T^{a}=\frac{1}{2} \sum_{A} \tau_{A}^{a}, \quad S^{i}=\frac{1}{2} \sum_{A} \sigma_{A}^{i}, \quad G^{i a}=\frac{1}{2} \sum_{A} \sigma_{A}^{i} \tau_{A}^{a} .
$$

The one-nucleon irreducible representations is a quartet made of a spin and isospin $\operatorname{doublet} \mathbf{4}=(p \uparrow$ $, p \downarrow, n \uparrow, n \downarrow)=(S=1 / 2, T=1 / 2)$. NN states with relative angular momentum $L$ and total spin $S$ and isospin $T$ fulfilling $(-1)^{S+L+T}=-1$ due to Fermi statistics correspond to an antisymmetric sextet and a symmetric decuplet which, in terms of $(S, T)$ representations of the $S U_{S}(2) \otimes S U_{T}(2)$ subgroup, are

$$
\begin{array}{rlll}
\mathbf{6}_{A} & =(1,0) \oplus(1,0) & L=0,2, \ldots & \rightarrow\left({ }^{1} S_{0},{ }^{3} S_{1}\right),\left({ }^{1} D_{2},{ }^{3} D_{1,2,3}\right),\left({ }^{1} G_{2},{ }^{3} G_{1,2,3}\right), \ldots \\
\mathbf{1 0}_{S}=(0,0) \oplus(1,1) & L=1,3, \ldots & \rightarrow & \left({ }^{1} P_{1},{ }^{3} P_{0,1,2}\right),\left({ }^{1} F_{1},{ }^{3} F_{0,1,2}\right), \ldots
\end{array}
$$

In particular, one obtains $V_{{ }^{3} S_{1}}(r)=V_{1_{S_{0}}}(r)$ which seems verified for $r>2 \mathrm{fm}$ (but not below). An amazing result is the large $N_{c}$ justification of this symmetry to $\mathscr{O}\left(1 / N_{c}^{2}\right)$ accuracy $[4,5]$ which strongly suggests to understand in what sense can the symmetry be ckecked in the much studied $\mathrm{NN}$ interaction, as this is a direct consequence of the underlying QCD dynamics.

A long distance interpretation of the symmetry has been given within a large $N_{c}$ spirit recently, particularly the role in higher partial waves and the companion Serber symmetry $[6,7,8]$. Within a Wilsonian approach saturation of effective parameters has been observed in [9] and in [10] by two different methods. On a more fundamental level, recent lattice calculations have observed Wigner's symmetry at the potential level [11] and also at the scattering length level for the unphysical pion masses about four times larger than in the real world [12]. In the present contribution we summarize the findings of our renalysis [13] based on the Similarity Renormalization Group and provide some outlook.

\section{Wigner Symmetry and Potentials}

A rather simple way to see how Wigner symmetry emerges from low energy NN-scattering data is by taking as an effective interaction a square well potential of depth $-V_{0}$ and range $r_{c}$. The potential parameters will be fixed by the corresponding scattering length $\alpha_{0}$ and effective range $r_{0}$ given by the equations,

$$
\alpha_{0}=r_{c}-\frac{\tan \sqrt{M V_{0}} r_{c}}{\sqrt{M V_{0}}}, \quad r_{0}=r_{c}\left[1-\frac{1}{\alpha_{0} r_{c} M V_{0}}-\frac{r_{c}^{2}}{3 \alpha_{0}^{2}}\right] .
$$



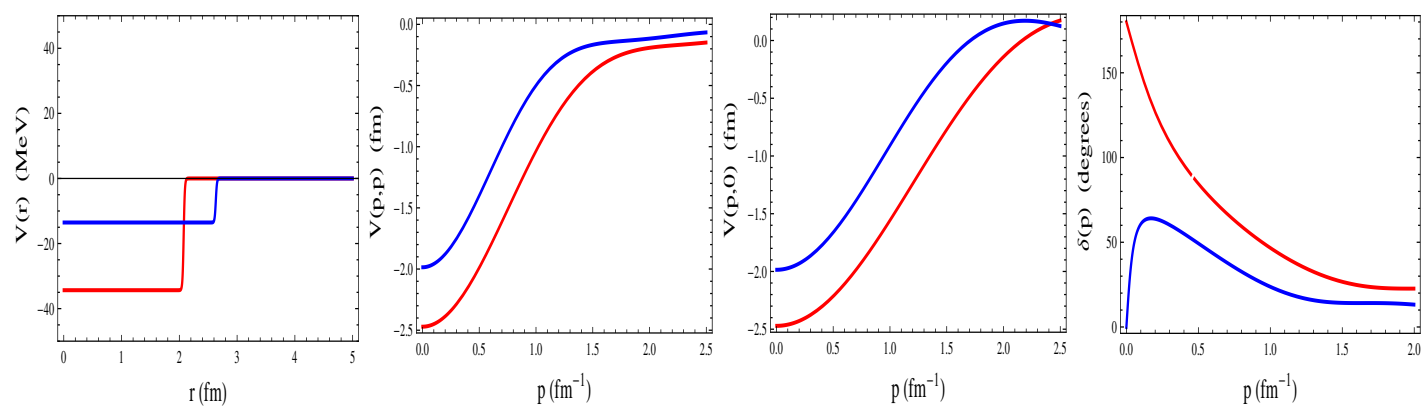

Figure 1: Square wells potentials, diagonal matrix elements, $V(p, p)$, off-diagonal matrix elements, $V(0, p)$, and phase shifts for the ${ }^{1} S_{0}$ (blue) and ${ }^{3} S_{1}$ (red) channels. Parameters are adjusted to reproduce the scattering lengths and effective ranges (see main text).

We will, in addition, define the volume integrals of the potential as

$$
C_{0}=\int d^{3} x V_{\mathrm{eff}}(\vec{x})=-\frac{4 \pi}{3} V_{0} r_{c}^{3}, \quad C_{2}=-\frac{1}{6} \int d^{3} x r^{2} V_{\mathrm{eff}}(\vec{x})=-\frac{4 \pi}{30} V_{0} r_{c}^{5}
$$

Now fixing the values in the ${ }^{1} S_{0}\left(\alpha_{0}=-23.74 \mathrm{fm}, r_{0}=2.75 \mathrm{fm}\right)$ and ${ }^{3} S_{1}\left(\alpha_{0}=5.40 \mathrm{fm}, r_{0}=\right.$ $1.75 \mathrm{fm}$ )channels, and imposing that there are no ${ }^{1} S_{0}$ bound states and just one ${ }^{3} S_{1}$ bound state (the deuteron) we get $\left(C_{0,{ }^{1} S_{0}}, C_{0,{ }^{3} S_{1}}\right)=(-1030,-1288) \mathrm{MeVfm}^{3}$ and $\left(C_{2,{ }^{1} S_{0}}, C_{2,{ }^{3} S_{1}}\right)=(715,554) \mathrm{MeVfm}^{5}$. The numerical value for $C_{0}$ agrees with the EFT estimate [14]. The role of tensor force and higher partial waves has been further explored [9]. Note that the volume integrals as well as the momentum space potential generically defined as

$$
V_{l^{\prime}, l}^{J S}\left(p^{\prime}, p\right)=M_{N} \int_{0}^{\infty} j_{l^{\prime}}\left(p^{\prime} r\right) j_{l}(p r) V_{l^{\prime} l}^{J S}(r) r^{2}
$$

are very similar numerically (see Fig. 1), $V_{3} S_{1} \sim V^{{ }_{1}} S_{0}$. For the phase-shifts the previous approximation is crude but it makes sense for wavelengths larger than the range of the NN interaction when compared to high quality potentials [15].

\section{Wigner Symmetry and the SRG}

The previous analyses of the effective interaction and Wigner symmetry are based on very low energy data. There is another rather surprising way of unveiling the Wigner symmetry beyond this restricted range and can be seen by using data up to about the lowest pion production inelastic threshold. The SRG method has been amply used for NN interactions in the last years $([17,18])$ and is based an an integro-differential equation for every (coupled) partial waves

$$
-\frac{1}{4} \lambda^{5} \frac{d V_{\lambda}\left(p^{\prime}, p\right)}{d \lambda}=-\left(p^{2}-p^{\prime 2}\right)^{2} V_{\lambda}\left(p^{\prime}, p\right)+\frac{2}{\pi} \int_{0}^{\infty} q^{2} d q\left(p^{2}+p^{\prime 2}-2 q^{2}\right) V_{\lambda}\left(p^{\prime}, q\right) V_{\lambda}(q, p(3) .1)
$$

where $\lambda$ is the SRG cut-off. The solution generates from an initial potential $V_{\lambda=\infty}\left(p^{\prime}, p\right)$ a oneparameter family of phase-equivalent potentials at all energies, $\delta_{\lambda}(p)=\delta_{\lambda=\infty}(p)$ which are driven to a stable fixed point at $\lambda \rightarrow 0$ [13]. Large momentum-differences $\left|p-p^{\prime}\right| \gg \lambda$ are suppressed as

$$
V_{\lambda}\left(p^{\prime}, p\right) \approx V_{\lambda=\infty}\left(p^{\prime}, p\right) e^{-\left(p^{2}-p^{\prime 2}\right)^{2} / \lambda^{4}}+\ldots
$$



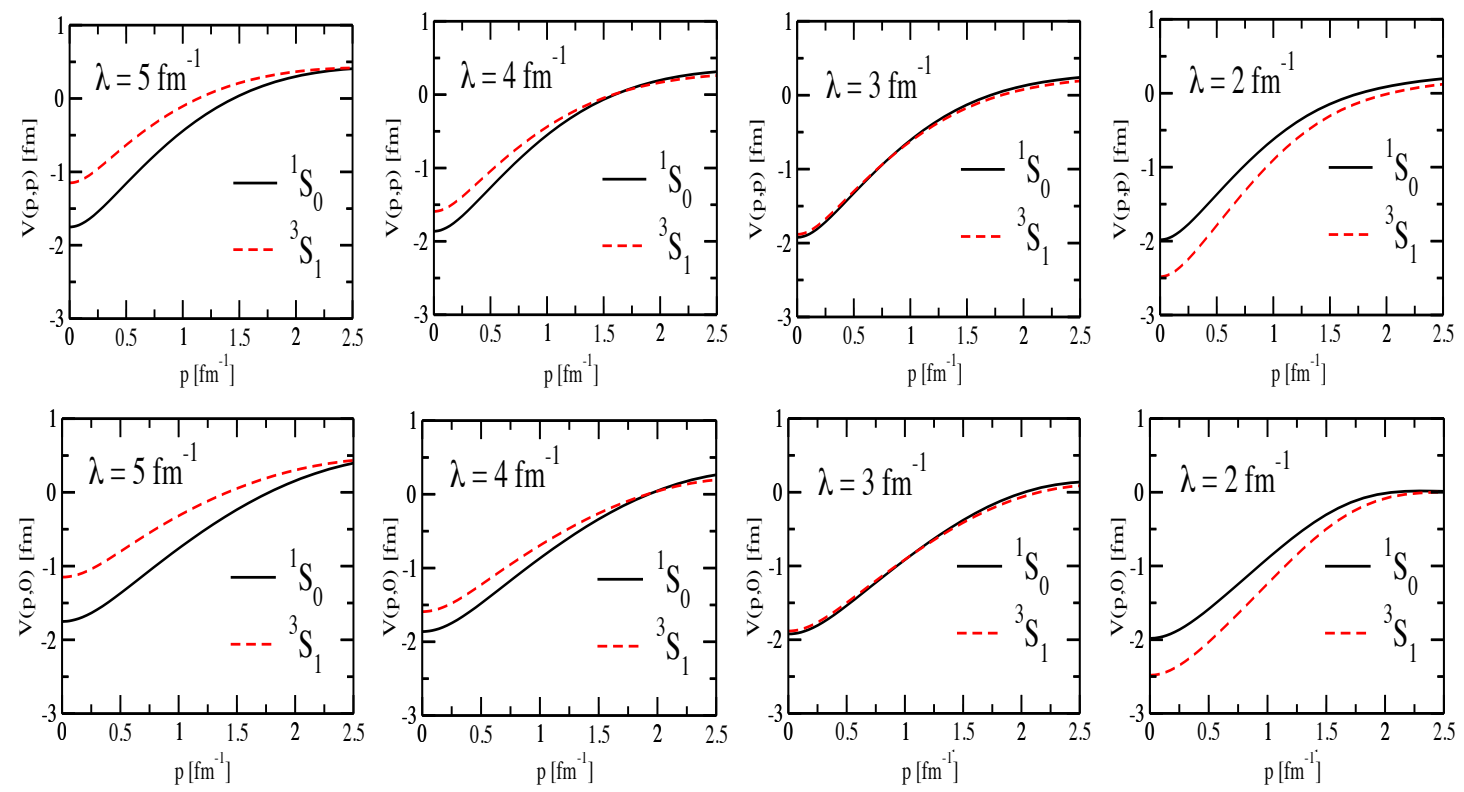

Figure 2: Comparison between diagonal, $V(p, p)$, and fully off-diagonal, $V(p, 0)$, matrix-elements of the SRG-evolved potentials for the $S$-waves (in $\mathrm{fm}$ ) as a function of the CM momentum $p$ (in $\mathrm{fm}^{-1}$ ), showing Wigner symmetry for $\lambda_{\text {Wigner }} \approx 3 \mathrm{fm}^{-1}$. We use the Argonne AV18 potential as the initial condition [16].

implying a simplification of the nuclear many body problem. We show in Fig. 2 (compare with Fig. 1) the results for the ${ }^{1} S_{0}$ and ${ }^{3} S_{1}$ channels for several SRG-cut-offs for the AV18 potential [16]. As we can see, there is a scale $\lambda_{\text {Wigner }} \sim 3 \mathrm{fm}^{-1}$ where

$$
V^{{ }^{S_{0}}, \lambda_{\text {Wigner }}}{ }\left(p^{\prime}, p\right) \approx V_{S_{1}, \lambda_{\text {Wigner }}}\left(p^{\prime}, p\right)
$$

Of course, since the SRG transformation is unitary, the phase-shifts remain the same for any value of $\lambda$ and despite the ${ }^{1} S_{0}$ and ${ }^{3} S_{1}$ phase-shifts being very different the SRG-evolved and phaseequivalent potentials look very similar.

\section{Finite Nuclei and Nuclear matter}

If is tempting to make simple estimates based on harmonic oscillator (HO) shell model wave functions. Of course, once we make a unitary transformation such as SRG on the two-body sector we are effectively generating multinucleon forces [19]. From a practical point of view, 3- and 4-nucleon forces are so far fixed from ${ }^{3} \mathrm{H}$ or ${ }^{4} \mathrm{He}$ binding energies, and they turn out to almost vanish at about $\lambda \sim 2 \mathrm{fm}^{-1}[19]$. From Fig. 3 we see that for $\lambda \sim 1 \mathrm{fm}^{-1}$ we get $\left(B_{d}^{\mathrm{HO}}, B_{3 \mathrm{H}}^{\mathrm{HO}}, B_{4 \mathrm{He}}^{\mathrm{HO}}\right)=$ $(-1.5,-7.1,-24.0) \mathrm{MeV}$, close (except for $\left.B_{d}\right)$ to more accurate calculations [19], regardless on using NijmII [15], AV18 [16] and the chiral N3LO-EM [21] and N3LO-EGM [22] potentials. This is in line with the coarse grained potentials calculation [20] and are marginal, at least for the ${ }^{3} \mathrm{H}$ and ${ }^{4} \mathrm{He}$ binding. Further, working at the SRG Wigner scale $\lambda_{\text {Wigner }}=3 \mathrm{fm}^{-1}$ gives unbound triton and a poor value $B_{4 \mathrm{He}}^{\mathrm{HO}}=-10 \mathrm{MeV}$, while in Ref. [19] it is found $\left(B_{3 \mathrm{H}}, B_{4 \mathrm{He}}\right)=(-8.1,-26.8) \mathrm{MeV}$. Nuclear matter in the Hartree-Fock approximation saturates, although it describes a Coester-like 

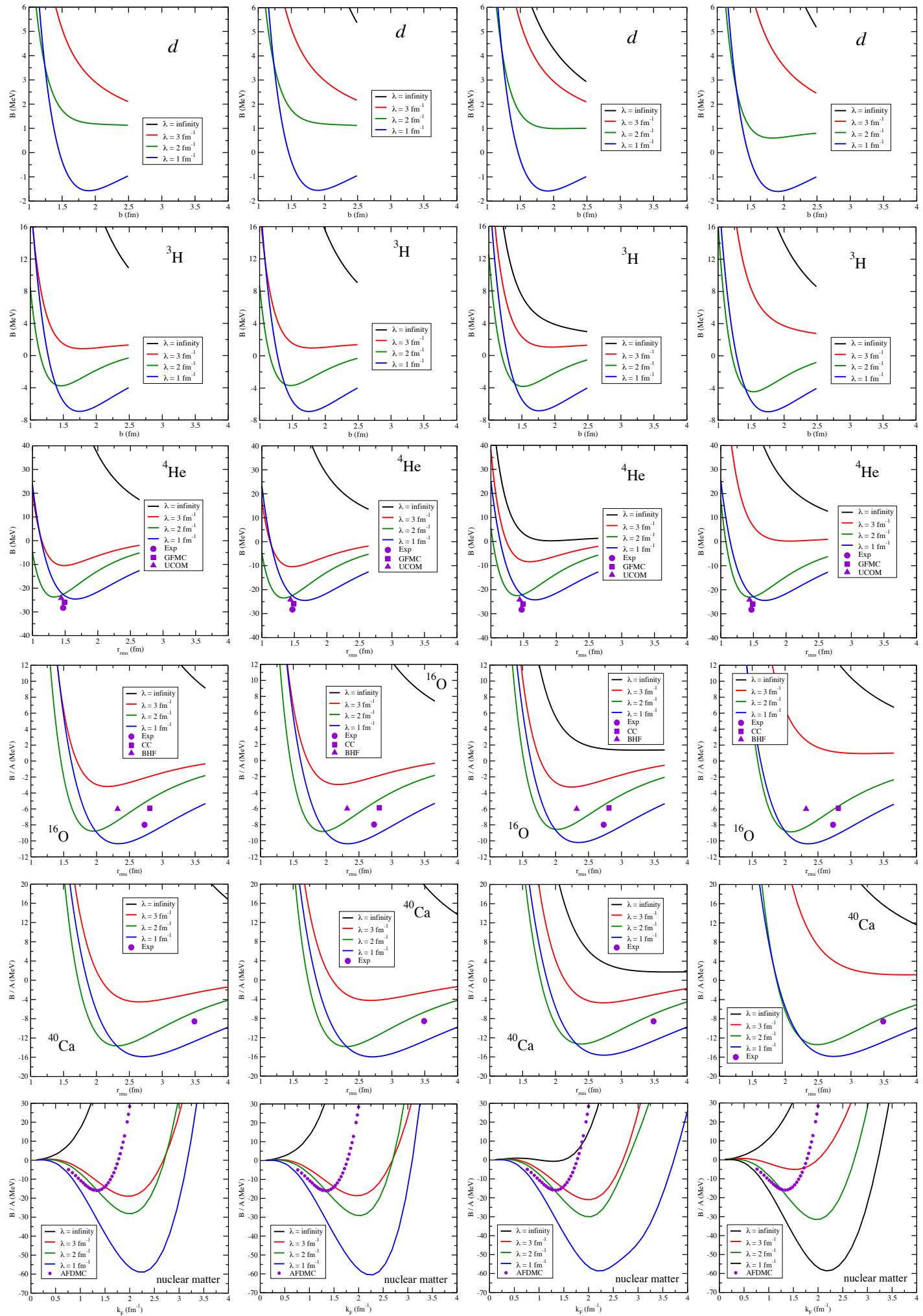

Figure 3: Binding energies including Coulomb (in MeV) vs msr $r_{m}$ (in fm) for ${ }^{2} \mathrm{H}=d,{ }^{3} \mathrm{H},{ }^{4} \mathrm{He},{ }^{16} \mathrm{O}$ and ${ }^{40} \mathrm{Ca}$ and nuclear matter for different SRG- $\lambda$ for NijmII [15], AV18 [16], N3LO-EM [21] and N3LOEGM [22] potentials (from left to right) and Harmonic Oscillator wave functions. We compare to some calculations and also to experimental data. Nuclear matter is computed in the Hartree-Fock approximation. 
band along the SRG trajectory typical of two-body interacions. This of course raises the question on whether or not the needed 3- and 4-body forces are SU(4)-invariant (see e.g.[23]).

\section{Conclusions}

From a fundamental viewpoint, QCD large $N_{c}$ based arguments foresee fulfilling Wigner symmetry with a relative $\mathscr{O}\left(N_{c}^{-2}\right)$ accuracy. This complies with our finding at $\lambda_{\text {wigner }}$, a remarkable and surprissing result if we consider that nowhere in the design and optimization of the modern high-quality interactions was the Wigner symmetry pattern explicitly implemented.

\section{References}

[1] L. Coraggio, A. Covello, A. Gargano, N. Itaco and T. T. S. Kuo, Prog. Part. Nucl. Phys. 62 (2009) 135

[2] S. K. Bogner, R. J. Furnstahl and A. Schwenk, Prog. Part. Nucl. Phys. 65, 94 (2010)

[3] E. Wigner. Phys.Rev., 51:106-119, 1937.

[4] David B. Kaplan and Martin J. Savage. Phys.Lett., B365:244-251, 1996.

[5] David B. Kaplan and Aneesh V. Manohar. Phys.Rev., C56:76-83, 1997.

[6] A. Calle Cordon and E. Ruiz Arriola. Phys.Rev., C78:054002, 2008.

[7] A. Calle Cordon and E. Ruiz Arriola. Phys.Rev., C80:014002, 2009.

[8] E. Ruiz Arriola and A. Calle Cordon. PoS, EFT09:046, 2009.

[9] E. Ruiz Arriola, arXiv:1009.4161 [nucl-th].

[10] R. Navarro Perez, J. E. Amaro and E. Ruiz Arriola, arXiv:1209.6269 [nucl-th].

[11] N. Ishii, S. Aoki, and T. Hatsuda. Phys.Rev.Lett., 99:022001, 2007.

[12] S. R. Beane, E. Chang, S. D. Cohen, W. Detmold, P. Junnarkar, H. W. Lin, T. C. Luu and K. Orginos et al., arXiv:1301.5790 [hep-lat].

[13] V.S. Timoteo, S. Szpigel, and E. Ruiz Arriola. Phys.Rev., C86:034002, 2012.

[14] Thomas Mehen, Iain W. Stewart, and Mark B. Wise. Phys.Rev.Lett., 83:931-934, 1999.

[15] V. G. J. Stoks, R. A. M. Klomp, C. P. F. Terheggen and J. J. de Swart, Phys. Rev. C 49 (1994) 2950

[16] Robert B. Wiringa, V. G. J. Stoks, and R. Schiavilla. Phys. Rev., C51:38-51, 1995.

[17] S. K. Bogner, R. J. Furnstahl, and R. J. Perry. Phys. Rev., C75:061001, 2007.

[18] Sergio Szpigel, Varese S. Timoteo, and Francisco de O. Duraes. Annals Phys., 326:364-405, 2011.

[19] E. D. Jurgenson, P. Navratil, and R. J. Furnstahl. Phys. Rev. Lett., 103:082501, 2009.

[20] R. Navarro Perez, J.E. Amaro, and E. Ruiz Arriola. Prog.Part.Nucl.Phys., 67:359-364, 2012.

[21] D. R. Entem and R. Machleidt. Phys. Rev., C68:041001, 2003.

[22] E. Epelbaum, W. Glockle, and Ulf-G. Meissner. Nucl.Phys., A747:362-424, 2005.

[23] M. C. Birse, B. Krippa and N. R. Walet, arXiv:1207.5735 [nucl-th]. 